\title{
PREDICTORS OF FAMILY AND MARITAL FUNCTIONING AT EARLY POSTPARTUM
}

\author{
Fei-Wan Ngai \\ The University of Hong Kong \\ Pokfulam, Hong Kong
}

\begin{abstract}
Achieving positive family and marital functioning has become an important issue in perinatal health because it is vital to the child's development and well-being. The purpose of this study was to examine the predictive role of family sense of coherence, social support and stress during pregnancy on family and marital functioning among Chinese mothers at six weeks postpartum.
\end{abstract}

\section{Methods}

This study used a longitudinal design. A convenience sample of 202

Chinese childbearing women completed assessment during pregnancy and at six weeks postpartum. The Medical Outcomes Study Family and Marital Functioning Measures, Family Sense of Coherence Scale, Medical Outcome Study Social Support Survey, and Social Readjustment Rating Scale were used to assess family and marital functioning, family sense of coherence, social support and stress, respectively.

\section{Results}

Multiple regression analysis showed that greater family sense of coherence, better social support and lower stress during pregnancy predicted higher family and marital functioning at six weeks postpartum. Changes in family sense of coherence and stress from pregnancy to postpartum also predicted family and marital functioning at six weeks postpartum.

\section{Conclusions}

The findings indicate that family sense of coherence, social support, and stress are important factors affecting family and marital functioning at early postpartum. Culturally competent healthcare should be developed to strengthen women's family sense of coherence and foster social support to reduce the stress of new motherhood, thereby promoting positive family and marital functioning.

Keywords: Family and marital functioning, family sense of coherence, social support, stress, perinatal period. 